\title{
Prevalência e evitabilidade de eventos adversos cirúrgicos em hospital de ensino do Brasil*
}

\author{
Josemar Batista ${ }^{1,2,3}$ \\ (D) https://orcid.org/0000-0001-9838-1232 \\ Elaine Drehmer de Almeida $\mathrm{Cruz}^{4}$ \\ (iD) https://orcid.org/0000-0002-7686-6340 \\ Francine Taporosky Alpendre ${ }^{1,5}$ \\ (D) https://orcid.org/0000-0003-1506-160X \\ Denise Jorge Munhoz da Rocha ${ }^{6}$ \\ (iD) https://orcid.org/0000-0002-2385-2391 \\ Marilise Borges Brandão 6 \\ (D) https://orcid.org/0000-0002-9090-8607 \\ Eliane Cristina Sanches Maziero ${ }^{1,7}$ \\ (D) https://orcid.org/0000-0003-1264-0833
}

* Artigo extraído da dissertação de mestrado "Avaliação da cultura de segurança e ocorrência de eventos adversos cirúrgicos em diferentes naturezas administrativas da gestão pública", apresentada à Universidade Federal do Paraná, Curitiba, PR, Brasil.

1 Universidade Federal do Paraná, Curitiba, PR, Brasil.

2 Faculdades Santa Cruz, Curitiba, PR, Brasil.

3 Bolsista da Coordenação de Aperfeiçoamento de Pessoal de Nível Superior (CAPES), Brasil.

${ }^{4}$ Universidade Federal do Paraná, Departamento de Enfermagem, Curitiba, PR, Brasil.

${ }^{5}$ Complexo Hospitalar de Clínicas da Universidade Federal do Paraná, Unidade de Centro Cirúrgico, Curitiba, PR, Brasil.

${ }^{6}$ Complexo Hospitalar de Clínicas da Universidade Federal do Paraná, Assessoria da Gestão da Qualidade, Curitiba, $\mathrm{PR}$, Brasil.

7 Governo do Estado do Paraná, Secretaria de Saúde do Estado do Paraná, Curitiba, PR, Brasil.
Objetivo: estimar a prevalência e evitabilidade de eventos adversos cirúrgicos em hospital de ensino e classificar os eventos segundo o tipo de incidente e grau do dano. Método: estudo transversal e retrospectivo realizado em duas fases. $\mathrm{Na}$ fase I, enfermeiros realizaram revisão retrospectiva em amostra aleatória simples de 192 prontuários de pacientes adultos, utilizando o formulário do Canadian Adverse Events Study para rastreamento de casos. A fase II objetivou a confirmação do evento adverso por comitê de especialistas composto por médico e enfermeiros. Os dados foram analisados por estatística descritiva univariada. Resultados: a prevalência de eventos adversos cirúrgicos foi de 21,8\%; em $52,4 \%$ dos casos, a detecção ocorreu no retorno ambulatorial. Dos 60 casos analisados, 90\% $(n=54)$ eram evitáveis e mais de dois terços resultaram em danos leves a moderados. Falhas técnicas cirúrgicas contribuíram em, aproximadamente, $40 \%$ dos casos. Houve prevalência da categoria infecção associada à atenção à saúde $(50 \% ; n=30)$. Destacaramse os eventos adversos relacionados à infecção de sítio cirúrgico $(30 \% ; n=18)$, deiscência de sutura $(16,7 \% ; n=10)$ e hematoma/seroma $(15 \% ; n=9)$. Conclusão: a prevalência e evitabilidade dos eventos adversos cirúrgicos são desafios a serem enfrentados pela gestão hospitalar.

Descritores: Segurança do Paciente; Erros Médicos; Doença Iatrogênica; Procedimentos Cirúrgicos Operatórios; Complicações Pós-Operatórias; Infecção da Ferida Cirúrgica.

\section{Como citar este artigo}

Batista J, Cruz EDA, Alpendre FT, Rocha DJM, Brandão MB, Maziero ECS. Prevalence and avoidability of surgical adverse events in a teaching hospital in Brazil. Rev. Latino-Am. Enfermagem. 2019;27:e2939. [Access Available in: DOI: http://dx.doi.org/10.1590/1518-8345.2939.3171. 


\section{Introdução}

A segurança e qualidade do cuidado perioperatório estão diretamente relacionadas ao desenvolvimento de modelos tecnoassistenciais, constituindo desafios para as organizações de saúde pela crescente evolução tecnológica, incorporação de novos processos clínicos e técnicas cirúrgicas. Esses avanços contribuem com a qualidade dos serviços prestados à sociedade ao mesmo tempo em que representam riscos assistenciais, os quais são agravados por falhas estruturais do sistema ou pela deficiência na gestão dos processos de trabalho(1), culminando na ocorrência de eventos adversos em pacientes submetidos ao tratamento cirúrgico.

A Organização Mundial de Saúde (OMS) define evento adverso (EA) como qualquer incidente que resultou em dano ao paciente ${ }^{(2)}$ e pressupõe que sejam realizadas 230 milhões de cirurgias ao ano no mundo, com ocorrência de sete milhões de EA e um milhão de pacientes evoluindo para óbito(3). Estimase que há potencial de evitabilidade em metade dos casos, nos quais a cirurgia leva a $\operatorname{danos}^{(3)}$, dados esses que fomentam a necessidade da adoção de práticas sistemáticas para o atendimento seguro do paciente no período perioperatório.

Revisão sistemática identificou taxa de EA cirúrgico de $14,4 \%{ }^{(4)}$, enquanto never events (eventos que nunca deveriam ocorrer) representaram, em pacientes cirúrgicos norte-americanos, a ocorrência de cirurgia em local errado e itens cirúrgicos retidos de 1 EA /100.000 e 1 EA/10.000 procedimentos, respectivamente ${ }^{(5)}$. No Brasil, apesar de não haver dados sistematizados, estudo pioneiro conduzido em três hospitais de ensino da região Sudeste, com dados de 1.103 internações do ano de 2003, encontrou incidência de EA de 7,6\%; entre estes, $35,2 \%$ foram atribuídos a procedimentos cirúrgicos ${ }^{(6)}$.

Os EA permanecem insuficientemente investigados mesmo consistindo em fator potencial de morbidade e custos econômicos(7), em especial os relacionados à assistência cirúrgica. Considera-se que estudar a ocorrência de EA cirúrgicos configura ferramenta gerencial que permite reconhecer, implantar e avaliar ações de melhoria, além de organizar e sistematizar os elementos que compõem a estrutura e o processo de trabalho em saúde.

Dessa forma, ao considerar a transição demográfica, epidemiológica e político-institucional em nível nacional e regional, destaca-se a importância de estudos nesse contexto como estratégia ao incentivo às ações preventivas. Essas ações devem estar em consenso com os resultados da 55a Assembleia Mundial de Saúde cujas metas são promover a segurança do paciente e a qualidade do atendimento em saúde ${ }^{(3)}$.
Diante do exposto, justifica-se a presente pesquisa que teve como pergunta norteadora: Qual a prevalência, evitabilidade e grau de dano dos EA cirúrgicos ocorridos em pacientes internados em hospital de ensino do Brasil? Assim, o objetivo desta pesquisa foi estimar a prevalência e evitabilidade de EA cirúrgicos em hospital de ensino do Brasil e classificá-los segundo o tipo de incidente e grau do dano.

\section{Método}

Estudo transversal e retrospectivo desenvolvido em um hospital público de ensino, de alta complexidade, localizado na região Sul do Brasil. O hospital conta com mais de 600 leitos financiados pelo Sistema Único de Saúde e realiza, em média, 840 cirurgias/mês. Em 2010 foi implantado o uso da Lista de Verificação de Segurança Cirúrgica proposta pelo protocolo "Cirurgias Seguras Salvam Vidas" da OMS. No decorrer do segundo semestre de 2014 foi instituído outro checklist, aplicado nas unidades de internação cirúrgica e complementar ao anterior, contendo 97 indicadores de segurança organizados em seis categorias: identificação, pré-operatório, pós-operatório imediato, pós-operatório mediato, outras complicações cirúrgicas e alta hospitalar ${ }^{(8)}$.

A população foi composta por prontuários de pacientes submetidos a procedimentos cirúrgicos internados nas unidades de ortopedia, cirurgia geral, cirurgia do aparelho digestivo, neurocirurgia, cirurgia plástica e transplante hepático, no período de junho de 2014 a maio de 2015. Analisou-se o primeiro procedimento cirúrgico, o qual correspondeu à internação analisada (internação índice) de pacientes com idade $\geq 18$ anos e com tempo de internação mínimo de 24 horas. Seguindo os critérios adotados por estudos prévios ${ }^{(9-10)}$ foram excluídos prontuários de pacientes psiquiátricos.

Foram elegíveis para o estudo 2.593 prontuários. Os parâmetros utilizados para definir o tamanho da amostra foram baseados a partir da incidência de complicações cirúrgicas de $16 \%{ }^{(3)}$, erro amostral de 5\% e nível de significância de $5 \%$ cujo cálculo resultou em 192 prontuários. A seleção aleatória foi realizada com base na lista de cirurgias emitida pelo serviço de informática da instituição. Os prontuários inelegíveis ou indisponíveis no serviço de arquivamento foram substituídos pelos prontuários imediatamente subsequentes da lista geral de cirurgias.

A identificação da ocorrência do EA e sua evitabilidade foi empregada por meio de revisão retrospectiva de prontuários com base em protocolo do Canadian Adverse Events Study - CAES que preconiza a identificação e análise do EA em duas fases ${ }^{(9)}$. A fase I se refere ao rastreamento de potenciais eventos adversos ( $p E A$ ) 
orientada por critérios explícitos, a qual foi executada por dupla revisão de prontuários por dois enfermeiros com experiência na área cirúrgica, utilizando-se o formulário de rastreamento traduzido e adaptado transculturalmente para o contexto brasileiro(10).

Esse formulário contempla 17 critérios de rastreamento de $\mathrm{pEA}$ relacionados aos procedimentos cirúrgicos e anestésicos, uso de medicamentos, diagnóstico, cuidados e tratamento não medicamentosos. Considerando a população desta pesquisa, foram excluídos os rastreadores relacionados ao aborto, trabalho de parto e parto, utilizando-se 16 rastreadores provenientes da lista original(9-10). Para a identificação dos pEA relacionados à infecção de sítio cirúrgico (ISC) ocorridos após a alta hospitalar, utilizaram-se os registros contidos nas fichas de consultas ambulatoriais e consideraram-se os critérios recomendados pelo Centers for Disease Control and Prevention que define ISC como aquela que ocorre em até 30 dias após o procedimento cirúrgico e/ou 90 dias após inserção de implante ${ }^{(11)}$.

Ao detectar a presença de, pelo menos, um critério de rastreamento, independente de qual rastreador, foi preenchido roteiro semiestruturado para caracterização do perfil demográfico, clínico, cirúrgico e anestésico. Posteriormente, foi preenchida a ficha de investigação de $\mathrm{pEA}$ e incluído o prontuário para a revisão na fase II. Esta se refere à confirmação, ou não, dos EA, por revisão estruturada implícita, a qual foi realizada por um médico e dois enfermeiros com tempo de atuação superior a 20 anos na área de gestão da qualidade e segurança do paciente.

Esse grupo compôs o comitê de especialistas com o objetivo de julgar a ocorrência, ou não, do EA perante consenso norteado pela definição do termo pela $\mathrm{OMS}^{(2)}$ e com a utilização de duas escalas. A primeira escala para julgar se o EA foi causado pela assistência prestada ao paciente e a segunda para avaliar o grau de evitabilidade. As escalas possuem seis pontos, considerou-se como EA e com potencial de evitabilidade quando a pontuação alcançou $\geq 4$ pontos ${ }^{(6,9)}$. Os eventos cirúrgicos foram assim classificados: fortemente prevenível, potencialmente prevenível, potencialmente não prevenível e fortemente não prevenível(6,9).

Os EA foram classificados, de acordo com o grau de dano físico, em leve, moderado, grave e óbito, e agrupados segundo a Classificação Internacional para a Segurança do Paciente na classe 1 (tipo de incidente), constituída pelas seguintes categorias: administração clínica; processo/ procedimento clínico; documentação; infecção relacionada à assistência à saúde (IRAS); medicação/fluidos endovenosos; sangue/hemoderivados; nutrição; oxigênio/ gases/vapores; dispositivos/equipamentos médicos; comportamento; acidentes com o paciente; infraestrutura/ local/instalações; e recursos/gestão organizacional(2).
As medidas utilizadas foram prevalência de EA cirúrgico entre os pacientes internados [(número de pacientes com pelo menos um EA cirúrgico/número total de pacientes) $\times$ 100] e proporção de EA cirúrgicos evitáveis [(número de EA cirúrgicos evitáveis/número total de EA cirúrgicos) x 100]. Os dados coletados foram transferidos para uma planilha do Microsoft Office Excel $2016^{\circledR}$ por dupla digitação para validação e checagem de sua consistência. Realizou-se a análise estatística descritiva univariada, empregando-se o software IBM SPSS 20 (Statistical Package for the Social Sciences).

Esta pesquisa pertence ao projeto temático "Avaliação da cultura de segurança e ocorrência de eventos adversos cirúrgicos em hospitais brasileiros", atendeu aos preceitos éticos de pesquisa envolvendo seres humanos e foi aprovada pelo Comitê de Ética em Pesquisa institucional, sob número 1.990.760.

\section{Resultados}

A frequência dos prontuários com rastreamento positivo para pEA e a taxa de prevalência e evitabilidade dos casos estão apresentadas na Figura 1. Dentre os 42 pacientes cirúrgicos acometidos por EA, 26,2\% $(n=11)$ tiveram mais de uma ocorrência, totalizando 60 EA cirúrgicos, dos quais $90 \%(n=54)$ foram classificados como preveníveis.

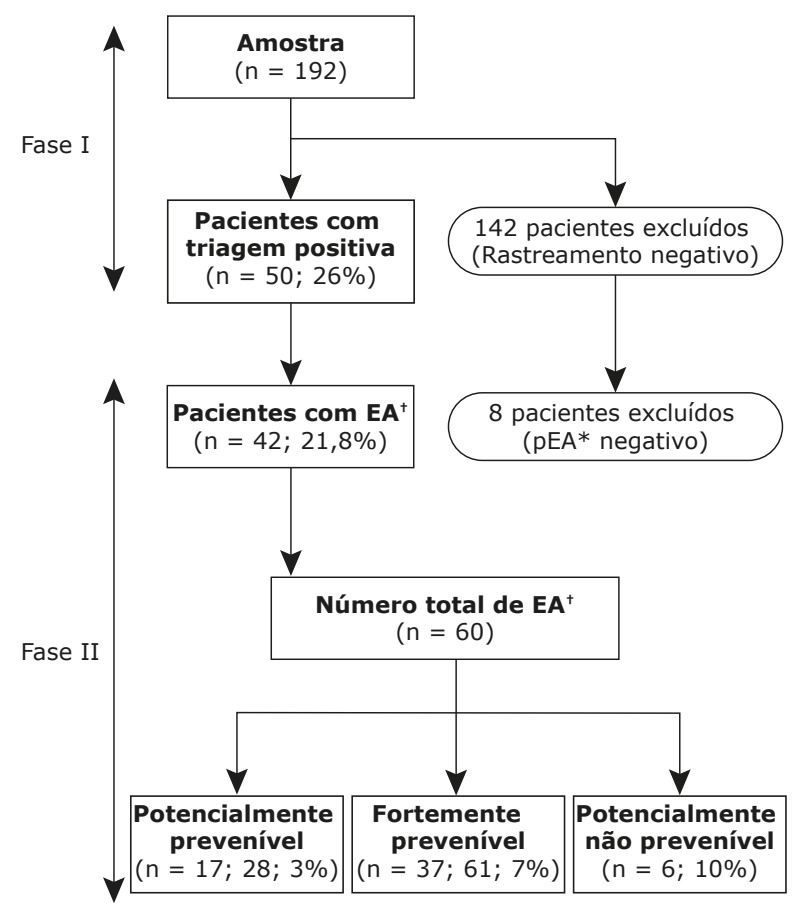

*pEA - potencial evento adverso; ${ }^{\dagger}$ EA- evento adverso.

Figura 1 = Fluxograma de seleção dos registros analisados e estimativa da prevalência e evitabilidade dos eventos adversos cirúrgicos. Curitiba, PR, Brasil, 2017 
Entre os pacientes cirúrgicos que apresentaram EA, a média de idade foi de 44,5 anos (desvio padrão - DP $\pm 15,5)$ e a média de internamento, de 11,9 dias (DP $\pm 21,1$ ), variando de um a 102 dias. As internações estiveram relacionadas a procedimentos cirúrgicos eletivos e, entre as condições clínicas, os pacientes apresentaram doença sistêmica leve. As comorbidades/fatores de riscos mais frequentes foram hipertensão arterial severa $(33,3 \%, n=14)$, tabagismo $(23,8 \%, \mathrm{n}=10)$, diabetes mellitus $(11,9 \%, \mathrm{n}=5)$ e obesidade $(9,5 \%, n=4)$. As demais características demográficas, cirúrgicas e anestésicas dos pacientes com EA cirúrgicos estão apresentadas na Tabela 1.

Tabela 1 - Características demográficas, cirúrgicas e anestésicas dos pacientes com eventos adversos cirúrgicos. Curitiba, PR, Brasil, 2017

\begin{tabular}{lcc}
\hline Variáveis & $\mathbf{n}$ & $\%$ \\
\hline Sexo & 24 & 57,1 \\
Masculino & 18 & 42,9 \\
Feminino & & \\
Faixa etária & 33 & 78,6 \\
$<60$ anos & 9 & 21,4 \\
$\geq 60$ anos & & \\
Tempo de internação pré-operatório & 37 & 88,1 \\
$<24$ horas & 5 & 11,9 \\
$\geq 24$ horas & & \\
Dias de internamento & 16 & 38,1 \\
1 a 2 dias & 9 & 21,4 \\
3 a 5 dias & 17 & 40,5 \\
$\geq 6$ dias & & 85,7 \\
Classificação cirúrgica & 36 & 14,3 \\
Eletiva & 6 & \\
Emergência & & \\
\hline
\end{tabular}

(continua...)
Tabela 1 - continuação

\begin{tabular}{lcc}
\hline Variáveis & $\mathbf{n}$ & $\%$ \\
\hline Grau de contaminação & & \\
$\quad$ Limpa & 16 & 38,1 \\
Potencialmente contaminada & 11 & 26,2 \\
Contaminada & 12 & 28,6 \\
$\quad$ Infectada & 3 & 7,1 \\
Duração da cirurgia & & \\
$\quad<120$ minutos & 22 & 52,4 \\
$\geq 120$ minutos & 20 & 47,6 \\
Risco cirúrgico ASA & & \\
ASA ${ }^{*}$ & & 16,7 \\
ASA ${ }^{*} \|^{\ddagger}$ & 7 & 61,9 \\
ASA ${ }^{*} I^{\S}$ & 26 & 21,4 \\
Tipo de Anestesia & 9 & \\
Raquianestesia & & 38,0 \\
Geral & 16 & 35,7 \\
Combinadall & 15 & 16,7 \\
Peridural & 7 & 4,8 \\
Local & 2 & 4,8 \\
\hline
\end{tabular}

*ASA = American Society of Anesthesiology $;{ }^{\dagger} \mathrm{I}=$ Paciente saudável; ${ }^{\ddagger} \mathrm{II}=$

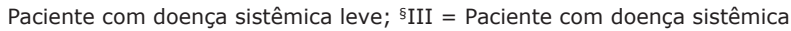
grave sem risco de morte; "Associação de duas ou mais tipos de anestesia

Os procedimentos cirúrgicos hernioplastia, artroplastia de joelho/quadril, apendicectomia e colecistectomia foram os que mais frequentemente evoluíram com EA $(47,6 \%$, $\mathrm{n}=20)$; cirurgias videolaparoscópicas representaram $14,3 \%$ dos casos $(n=6)$.

Os EA cirúrgicos concentraram-se na categoria infecção associada à atenção à saúde, com $50 \%$ dos casos $(n=30)$, seguidos de processo/procedimento clínico (38,4\%; $n=23)$, acidentes com o paciente $(8,3 \%$; $\mathrm{n}=5$ ) e dispositivo/equipamento médico, com 3,3\% $(n=2)$, conforme apresentado na Tabela 2. Quanto ao grau de dano físico aos pacientes que foram acometidos por EA cirúrgicos, $90 \%(n=54)$ foram classificados entre leve e/ou moderado. Nos registros analisados não foram detectados EA com desfecho de óbito.

Tabela 2 - Distribuição dos eventos adversos cirúrgicos segundo tipo de incidente, grau de dano e potencial de evitabilidade. Curitiba, PR, Brasil, 2017

\begin{tabular}{|c|c|c|c|c|c|c|c|c|}
\hline \multirow[t]{2}{*}{ Variável } & \multirow[b]{3}{*}{$\mathbf{n}$} & \multirow[b]{3}{*}{$\%$} & \multicolumn{3}{|c|}{ Grau de Dano } & \multicolumn{3}{|c|}{ Potencial de Evitabilidade } \\
\hline & & & Leve & Moderado & Grave & FE* $^{*}$ & $\mathrm{PE}^{\dagger}$ & PNE $^{\ddagger}$ \\
\hline Evento Adverso & & & n (\%) & $\mathrm{n}(\%)$ & $\mathrm{n}(\%)$ & n (\%) & $\mathrm{n}(\%)$ & n (\%) \\
\hline Infecção de Sítio Cirúrgico & 18 & 30,0 & $7(38,9)$ & $10(55,5)$ & $1(5,6)$ & $13(72,2)$ & $5(27,8)$ & $0(0,0)$ \\
\hline Deiscência & 10 & 16,7 & $7(70,0)$ & $3(30,0)$ & $0(0,0)$ & $8(80,0)$ & $1(10,0)$ & $1(10,0)$ \\
\hline Hematoma/Seroma & 9 & 15,0 & $5(55,6)$ & $3(33,3)$ & $1(11,1)$ & $7(77,8)$ & $2(22,2)$ & $0(0,0)$ \\
\hline Retenção Urinária & 5 & 8,4 & $5(100)$ & $0(0,0)$ & $0(0,0)$ & $0(0,0)$ & $0(0,0)$ & $5(100)$ \\
\hline Trombose Venosa Profunda & 3 & 5,0 & $0(0,0)$ & $3(100)$ & $0(0,0)$ & $0(0,0)$ & $3(100)$ & $0(0,0)$ \\
\hline Perfuração/Laceração & 3 & 5,0 & $0(0,0)$ & $2(66,6)$ & $1(33,3)$ & $2(66,6)$ & $1(33,3)$ & $0(0,0)$ \\
\hline Lesão de pele/mucosa & 2 & 3,3 & $1(50,0)$ & $1(50,0)$ & $0(0,0)$ & $2(100)$ & $0(0,0)$ & $0(0,0)$ \\
\hline Sepse/ Choque séptico & 2 & 3,3 & $1(50,0)$ & $1(50,0)$ & $0(0,0)$ & $1(50,0)$ & $1(50,0)$ & $0(0,0)$ \\
\hline Fístula & 2 & 3,3 & $0(0,0)$ & $2(100)$ & $0(0,0)$ & $0(0,0)$ & $2(100)$ & $0(0,0)$ \\
\hline Hemorragia & 2 & 3,3 & $0(0,0)$ & $1(50,0)$ & $1(50,0)$ & $1(50,0)$ & $1(50,0)$ & $0(0,0)$ \\
\hline Queda & 2 & 3,3 & $1(50,0)$ & $0(0,0)$ & $1(50,0)$ & $2(100)$ & $0(0,0)$ & $0(0,0)$ \\
\hline Hipóxia & 1 & 1,7 & $0(0,0)$ & $0(0,0)$ & $1(100)$ & $1(100)$ & $0(0,0)$ & $0(0,0)$ \\
\hline Rouquidão & 1 & 1,7 & $1(100)$ & $0(0,0)$ & $0(0,0)$ & $0(0,0)$ & $1(100)$ & $0(0,0)$ \\
\hline Total & 60 & 100 & $28(46,7)$ & $26(43,3)$ & $6(10,0)$ & $37(61,7)$ & $17(28,3)$ & $6(10,0)$ \\
\hline
\end{tabular}

*FE = Fortemente evitável; ${ }^{+} \mathrm{PE}=$ Potencialmente evitável; ${ }^{\ddagger} \mathrm{PNE}=$ Potencialmente não evitável 
Os registros do retorno ambulatorial contribuíram para $52,4 \%(n=22)$ dos casos e, do total destes pacientes, dois $(4,8 \%)$ foram reinternados em decorrência do EA.

\section{Discussão}

A revisão retrospectiva de prontuários, apesar das limitações próprias à técnica, permitiu identificar a prevalência de EA cirúrgicos, potencialmente evitáveis, em um único ambiente hospitalar. Os resultados, ora apresentados, suscitam a reflexão acerca da possível magnitude do problema no país, em especial em regiões menos privilegiadas economicamente, ao considerar as desigualdades geográficas e regionais no fornecimento de atendimento cirúrgico, bem como a disponibilidade de profissionais qualificados ${ }^{(12)}$.

A prevalência de $21,8 \%$ de EA cirúrgico encontrada na presente pesquisa foi superior à registrada em investigação conduzida na Suécia $(15,4 \%)^{(13)}$, em hospital universitário do Japão $(15,1 \%)^{(14)}$, e fica aquém de estudo realizado na Espanha com pacientes submetidos à cirurgia geral $(36,8 \%)^{(15)}$. No Brasil, em estudo realizado em três hospitais da região Sudeste, a incidência de EA cirúrgico foi de $3,5 \%{ }^{(16)}$, enquanto na Europa, estudo em 30 hospitais públicos de cuidados agudos constatou a incidência de $13,1 \%{ }^{(17)}$.

A literatura aponta que o desempenho dos revisores pode ser um dos fatores relacionados à subestimação de $\operatorname{casos}^{(18)}$. Entretanto, a frequência de pEA identificada na presente pesquisa apresentou similaridade com o desempenho de revisores cuja revisão primária alcançou $21,6 \%$ de rastreamento positivo(18) e ficou aquém de revisores suecos que encontraram $34,3 \%$ de prontuários positivos com pEA para inclusão na fase $\mathrm{II}^{(19)}$. Um dos fatores para a ocorrência da subestimação dos rastreadores concentrou-se, nesta pesquisa, em anotações/registros incompletos, ilegíveis e rasurados, fato possivelmente agravado pelo uso institucional de prontuário físico.

A evitabilidade dos EA cirúrgicos foi superior aos valores relatados em diversos estudos, com variação de $5,2 \%$ a $70,8 \%{ }^{(4,13,15-17,20)}$, o que suscita a necessidade de avaliar, concomitantemente, indicadores de assistência cirúrgica, e incita gestores, cirurgiões e profissionais da equipe de enfermagem, entre outros, a reavaliarem o processo assistencial e a proporem ações de melhoria contínua.

Observa-se, resguardadas as diferenças geográficas, os desenhos metodológicos utilizados em distintas pesquisas, e a qualidade dos serviços prestados em diferentes regiões e países do mundo, a evidência da vulnerabilidade dos pacientes à ocorrência de um, ou mais, EA cirúrgicos. Estes, majoritariamente evitáveis, conforme apontado por estudos prévios ${ }^{(13,16)}$ e reforçado pela presente pesquisa, a qual identificou que $26,2 \%$ dos pacientes $(n=11)$ sofreram mais de um EA durante a internação índice.

Esses achados revelam que erros e falhas no processo assistencial cirúrgico podem ocasionar diversos incidentes em um mesmo indivíduo, acarretando em danos físicos. Revisão sistemática mostrou que danos leves e moderados corresponderam a $86,7 \%$ dos casos $^{(4)}$. Dados esses compatíveis com os resultados aqui apresentados, em que mais de dois terços dos eventos resultaram em incapacidades leves a moderadas, reforçando a premissa do segundo desafio global em segurança do paciente (cirurgias seguras salvam vidas), da mesma forma que a utilização da lista de verificação de segurança cirúrgica pelos serviços de saúde contribui para a redução de EA no ambiente cirúrgico(3).

A instituição desta pesquisa implantou o checklist cirúrgico e também desenvolveu lista de verificação a ser aplicada nas unidades de internação, pela equipe de enfermagem, nos períodos pré e pós-operatório(8). Contudo, os resultados destacam EA cirúrgicos associados a falhas técnicas durante a execução do procedimento cirúrgico (hematoma/seroma, deiscência, perfuração/laceração, necrose de parede, hemorragia e embolia gasosa), contribuindo com aproximadamente $40 \%$ dos casos. Esse dado diverge de outro estudo que relatou EA relacionados a erros no manejo da internação em proporção superior que a técnica cirúrgica(4).

Desse modo, os resultadosapontam, principalmente, para a necessidade de revisão/aprimoramento da técnica operatória e são coerentes com os achados de outro estudo brasileiro, o qual apontou falhas técnicas em centro cirúrgico de $27 \%(n=7)^{(21)}$, do mesmo modo que em centro médico da China cujo estudo evidenciou $61,6 \%(n=16)$ dos EA relacionados a falhas técnicas e/ ou de vigilância(22).

Assim, por se tratar de um hospital de ensino, com profissionais em aprimoramento de habilidades clínicas e cirúrgicas, é imprescindível a capacitação e supervisão constantes. Isso com vistas a promover a qualidade da assistência cirúrgica e corrigir não conformidades. EA graves corresponderam, nesta pesquisa, a aproximadamente $10 \%$ dos casos, mostrando-se mais frequentes quando comparados à investigação norteamericana que analisou 676 EA cirúrgicos e encontrou prevalência de 6,36\% $(n=43)$ de eventos graves ${ }^{(20)}$. Em estudo brasileiro, 21,9\% ( $n=9)$ dos casos apresentaram danos permanentes, dos quais $17,1 \%(n=7)$ evoluíram para óbito ${ }^{(16)}$.

Dados de outras investigações apontaram que os EA são mais frequentes entre pacientes idosos ${ }^{(13,19)}$, diferentemente do ocorrido nesta investigação cuja 
maior prevalência foi entre pacientes na faixa etária entre 18 e 59 anos de idade. Fato esse que pode ser justificado, primeiramente, pelo perfil de atendimento das unidades cirúrgicas, prevalentemente composto por adultos jovens, baixa prevalência de doença sistêmica grave e ausência de fatores de risco/comorbidades. Soma-se o predomínio de cirurgias eletivas, nas quais é viável melhor preparo cirúrgico, bem como menor risco de incidentes relacionados à população mais jovem.

Os EA cirúrgicos estiveram relacionados à IRAS em $50 \%(n=30)$, e as ISC representaram quase um terço destes. Sabe-se que esses eventos são considerados os mais comuns entre os pacientes cirúrgicos, apesar das diversas estratégias baseadas em evidências que podem ser implementadas para sua redução(23), bem como a utilização da lista de verificação de segurança cirúrgica cuja adaptação ao contexto institucional foi

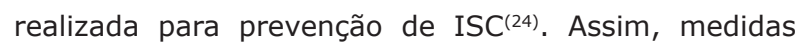
básicas e reconhecidas como evidência científica são recomendadas por instituições internacionais e corroboradas no Brasil, devendo fazer parte não somente de protocolos cirúrgicos, mas também de auditoria para a qualidade assistencial.

Outro fator a considerar na prevenção de ISC concentra-se na cultura de segurança da unidade, evidenciado em estudo transversal conduzido em sete hospitais americanos que associou os escores de cultura com a redução das taxas de ISC em cirurgias de cólon ${ }^{(25)}$. Diante da elevada prevalência de EA cirúrgicos relacionados à ISC, é necessário avaliar os indicadores de assistência cirúrgica que potencializam o risco de sua ocorrência ${ }^{(11,23)}$ e induzem à reflexão acerca da cultura de segurança e do desperdício financeiro no sistema de saúde brasileiro, ao se considerar que, majoritariamente, as ISC foram consideradas como fortemente ou potencialmente evitáveis.

A deiscência de sutura cirúrgica teve baixa prevalência em estudo americano, o qual analisou 676 EA cirúrgicos e encontrou dois $\operatorname{casos}^{(20)}$, porém representou $3,67 \%(n=8)$ dos EA em estudo nacional(26). Esses dados contrapõem os resultados desta pesquisa, na qual esse evento foi o segundo mais frequente, com prevalência de $16,7 \%(n=10)$, e indica a necessidade de avaliar, além da habilidade técnica profissional, a possibilidade de problemas técnicos com o material utilizado para a execução do procedimento.

O terceiro EA mais prevalente esteve associado a hematomas/seromas e representou, em estudo espanhol, $8,9 \%(n=16)$ de pacientes submetidos à cirurgia geral ${ }^{(15)}$. Esse EA se não tratado adequadamente pode causar desconforto físico e aumentar o risco de infecção(27), além de comprometer o processo de cicatrização e predispor os pacientes à deiscência de sutura de ferida operatória, cabendo um conjunto de ações relativas à técnica cirúrgica e cuidados pós-operatórios.

A trombose venosa profunda acometeu três pacientes $(5 \%)$, resultado superior ao identificado em estudo realizado no Japão $(1 \% ; n=3)^{(14)}$. Diversas são as medidas protetoras à ocorrência desse agravo e amplamente reconhecidas, desde identificação de pacientes de alto risco, profilaxia medicamentosa, deambulação precoce e uso de meias compressivas. É relevante o estabelecimento e seguimento rigoroso de protocolo de profilaxia de tromboembolismo, já que a literatura aponta que a incapacidade de implementar ou seguir um protocolo é fator contributivo para a ocorrência de $\mathrm{EA}^{(16,18)}$, tornando-se fator humano limitante para segurança do paciente e prevenção de EA cirúrgicos.

Referente à perfuração/laceração de órgão, observou-se prevalência de $5 \%(n=3)$ e foi associada, em sua maioria, à punção venosa de acesso central, evento potencialmente evitável com o uso de ultrassonografia durante o procedimento. Em hospital do Texas, Estados Unidos, as lacerações representaram 7,1\% ( $n=48)$, sendo $6,5 \%$ preveníveis, e quase a metade dos casos prolongaram a internação do paciente(20).

As lesões de pele e mucosa, seja por posicionamento cirúrgico, imobilidade no leito, reação alérgica a adesivos médico-hospitalares ou procedimentos clínicos, são passíveis de prevenção quando conduzida adequada abordagem pela equipe de saúde. Análise de 507 EA em unidades cirúrgicas de 63 hospitais da Suécia apontou que essas lesões acometeram 31 pacientes $(6,1 \%)$ e $94 \%$ foram considerados evitáveis ${ }^{(13)}$. Nesse sentido, é viável utilizar escala para estratificação do risco decorrente do posicionamento cirúrgico(28) como estratégia para minimizar sua ocorrência.

Casos de sepse/choque séptico representaram nesta pesquisa $3,3 \%$ dos EA, ficando aquém do encontrado no referido estudo da Suécia, com 13,2\% $(n=30)^{(13)}$. Por ser considerado grave e representar risco à vida do paciente, levanta-se a hipótese da capacitação dos profissionais para a identificação de sinais preditivos e diagnóstico precoce, tornando imperativo estudos para a determinação das causas, com vistas à prevenção.

Queda e hemorragia representaram prevalência de $3,3 \%(n=2)$ cada. Em estudo brasileiro, as hemorragias acometeram $12,2 \% \quad(n=5)^{(16)}$, enquanto que em hospitais de cuidados agudos dos Estados Unidos, com 676 pacientes, a prevalência foi de 35,6\% $(n=241)^{(20)}$. A baixa prevalência desse EA na presente pesquisa pode estar relacionada à imprecisão dos registros. Acreditase que a capacitação para a excelência na comunicação escrita e uso de instrumento padronizado para mensuração acurada poderão contribuir na identificação de casos e servir de base para condutas terapêuticas. 
As quedas tiveram prevalência de 2,7\% e 2,4\% dos pacientes cirúrgicos atendidos em unidade cirúrgica do Brasil e Suécia, respectivamente ${ }^{(13,26)}$. No hospital da presente pesquisa, há protocolo de prevenção de quedas, porém destaca-se a constante necessidade de aprimoramento, em especial pelo risco que representa ao paciente em pós-operatório.

Os EA considerados potencialmente não evitáveis foram, majoritariamente, relacionados à retenção urinária após o uso de opioide ou analgesia pósoperatória por cateter peridural. Estudo realizado em oito hospitais de cuidados agudos do Texas, Estados Unidos apontou que dos 40 EA cirúrgicos relacionados à retenção urinária, $92,5 \%(n=37)$ foram classificados como não evitáveis ${ }^{(20)}$.

A retenção urinária predispõe a riscos de infecção de trato urinário, uma vez que, frequentemente, exige terapêutica adicional, como cateterização vesical, além do risco pela retenção prolongada de urina que predispõe a proliferação microbiana. Contudo, tornase importante enfatizar que o tratamento agressivo da dor é fundamental, pois as consequências de um tratamento ineficaz da dor aguda são, muitas vezes, maiores do que o risco de efeitos colaterais adversos em decorrência do próprio uso do analgésico(29). Acredita-se que melhorar a avaliação pré-operatória pela equipe multidisciplinar e identificar os fatores de risco intrínsecos contribua para melhor planejamento pré-cirúrgico e redução desses casos.

Destaca-se também, como relevante nesta pesquisa, a alta detecção de EA por meio dos registros de retorno ambulatorial, com dois reinternamentos. É comprovado que os EA elevam o tempo de hospitalização, consequente aumento de custos hospitalares ${ }^{(7,16)}$, bem como retorno ambulatorial e intervenções em serviços de emergência precocemente. Esse achado reitera a necessidade de elaborar estratégias para vigilância cirúrgica pós-alta cujo objetivo é identificar eventos para além dos ambientes internos do hospital, inclusive com ativo sistema de notificação. Esses dados poderão subsidiar medidas preventivas, melhorar o diagnóstico da segurança do paciente, bem como o desenvolvimento progressivo da cultura de segurança organizacional, tornando-se elementos a serem gerenciados pelas unidades estudadas e pela organização hospitalar.

O estudo apresenta algumas limitações. Uma delas decorre de que os resultados são provenientes de revisão retrospectiva de prontuários de um único ambiente hospitalar, o que impede a generalização dos resultados da pesquisa. Houve incipiência na completude dos registros da equipe médica e de enfermagem, com potencial interferência na detecção dos EA; em alguns o desfecho óbito ocorreu em âmbito domiciliar e/ou outra instituição hospitalar, impossibilitando investigar o critério de rastreamento. Outro fator limitante foi a ausência de uniformidade em métodos de pesquisa e de classificação para detecção, análise e confirmação dos EA, os quais dificultam a comparação desses resultados entre diferentes contextos assistenciais de saúde.

Apesar dessas limitações, o estudo possui fortalezas. A primeira se concentra no fato da utilização de metodologia internacional padronizada para busca e confirmação de EA e da incipiência de estudos para estimar a prevalência de EA em população específica de pacientes cirúrgicos de hospital de ensino brasileiro. Além disso, o estudo é pioneiro no país ao investigar os EA cirúrgicos ocorridos durante a hospitalização e após a alta do paciente com retorno ambulatorial. Outro ponto forte está relacionado à fase de confirmação e análise dos EA, a qual foi conduzida por consenso de painel de especialistas, permitindo evitar o descarte indevido de prontuários rastreados e reduzir a subjetividade no julgamento dos casos.

\section{Conclusão}

Os achados evidenciaram que aproximadamente metade dos EA cirúrgicos foram identificados no retorno ambulatorial, causaram danos leves a moderados e, majoritariamente, foram classificados como evitáveis. Os EA cirúrgicos prevalentes foram as IRAS, com destaque para as ISC, que representaram quase um terço do total dos casos. A prevalência e evitabilidade dos EA cirúrgicos nesta pesquisa são desafios a serem enfrentados pela gestão hospitalar no contexto cirúrgico.

Espera-se que este estudo estimule a investigação da prevalência de EA cirúrgicos em diferentes contextos assistenciais e possa contribuir para a implementação de práticas seguras em saúde, com vistas à promoção da qualidade do cuidado, conforme recomendações internacionais e diretrizes nacionais.

\section{Referências}

1. Souza MRC, Russomano T. Experience in use of HFACS (Human Factors Analysis and Classification System) model in structuring causal maps of adverse events. Aviation in Focus. [Internet] 2017 [cited Jul 29, 2018]; 8(1):14-8. Available from: http://dx.doi.org/ 10.15448/2179-703X.2017.1.27186

2. World Health Organization. The Conceptual Framework for the International Classification for Patient Safety. 2009. [cited Jul 21, 2016]. Available from: http://www. who.int/patientsafety/taxonomy/icps_full_report.pdf

3. World Health Organization. World alliance for patient safety. The second Global Patient safety challenge. 
Safe surgery saves lives. Geneva; 2008. [cited Mar 25, 2016]. Available from: http://apps.who.int/iris/ handle/10665/70080

4. Anderson Oliver S, Davis R, Hanna GB, Vincent CA. Surgical adverse events: a systematic review. Am J Surg. [Internet] 2013 [cited Dec 30, 2017]; 206:2. Available from: https://doi.org/10.1016/j.amjsurg.2012.11.009 5. Hempel S, Maggard-Gibbons M, Nguyen DK, Dawes AJ, Miake-Lye I, Beroes JM, et al. Wrong-Site Surgery, Retained Surgical Items, and Surgical Fires A Systematic Review of Surgical Never Events. JAMA Surg. [Internet] 2015 [cited Dec 29, 2017]; 150:8. Available from: https://doi.org/10.1001/jamasurg.2015.0301

6. Mendes W, Martins M, Rozenfeld S, Travassos C. The assessment of adverse events in hospitals in Brazil. Int J Qual Health Care. [Internet] 2009 [cited Oct 4, 2016]; 21:4. Available from: http://dx.doi.org/10.1093/intqhc/ mzp022

7. Sousa-Pinto B, Marques B, Lopes F, Freitas A. Frequency and Impact of Adverse Events in Inpatients: A Nationwide Analysis of Episodes between 2000 and 2015. J Med Syst. [Internet] 2018 [cited Apr 1, 2018]; 42:3. Available from: http://dx.doi.org/10.1007/ s10916-018-0898-5

8. Alpendre FT, Cruz EDA, Dyniewicz AM, Mantovani MF, Silva AEBC, Santos GS. Safe surgery: validation of pre and postoperative checklists. Rev. LatinoAm. Enfermagem. [Internet] 2017 [cited Oct 29, 2017]; 25:e2907. Available from: http://dx.doi.org/ 10.1590/1518-8345.1854.2907

9. Baker GR, Norton PG, Flintoft V, Blais R, Brown A, Cox J, et al. The Canadian Adverse Events Study: the incidence of adverse events among hospital patients in Canada. CMAJ. [Internet] 2004 [cited Oct 4, 2017]; 170:11. Available from: https://doi.org/10.1503/ cmaj.1040498

10. Mendes W, Travassos C, Martins M, Marques PM. Adjustment of adverse events assessment forms for use in Brazilian hospitals. Rev Bras Epidemiol. [Internet] 2008 [cited Oct 7, 2016]; 11:1. Available from: http:// dx.doi.org/10.1590/S1415-790X2008000100005

11. Centers for Disease Control and Prevention; The National Healthcare Safety Network. Surgical Site Infection (SSI) Event. Manual: patient safety component manual [Internet]. Atlanta: CDC; 2017 [cited Feb 26, 2018]. Available from: https://www.cdc.gov/nhsn/pdfs/ pscmanual/pcsmanual_current.pdf

12. Moura MLO, Santos Diego LA. Lack of access to surgery: a public health problem. Cad Saúde Pública. [Internet] 2017 [cited Jan 19, 2018]; 33:10. Available from: http://dx.doi.org/10.1590/0102-311x00151817

13. Nilsson L, Risberg MB, Montgomery A, Sjodahl R, Schildmeijer K, Rutberg H. Preventable Adverse Events in Surgical Care in Sweden: A Nationwide Review of Patient Notes. Medicine. [Internet] 2016 [cited Jan 10, 2018]; 95:11. Available from: https://doi.org/10.1097/ MD.0000000000003047

14. Shiozaki K, Morimatsu H, Matsusaki T, Matsusaki T, Iwasaki T. Observational Study to Assess and Predict Serious Adverse Events after Major Surgery. Acta Med Okayama. [Internet] 2016 [cited Jan 19, 2018]; 70:6. Available from: http://dx.doi.org/10.18926/AMO/54809 15. Zapata AIP, Samaniego MG, Cuéllar ER, Cámara AGL, López PR. Comparison of the "Trigger" tool with the minimum basic data set for detecting adverse events in general surgery. Rev Calid Asist. [Internet] 2017 [cited Feb 12, 2018]; 32:4. Available from: https://doi.org/ 10.1016/j.cali.2017.01.001

16. Moura MLO, Mendes W. Assessment of surgical adverse events in Rio de Janeiro hospitals. Rev Bras Epidemiol. [Internet] 2012 [cited Apr 7, 2016]; 15:3. Available from: http://dx.doi.org/10.1590/ S1415-790X2012000300007

17. Rafter N, Hickey A, Conroy RM, Condell S, O ' Connor $P$, Vaughan $D$, et al. The Irish National Adverse Events Study (INAES): the frequency and nature of adverse events in Irish hospitals - a retrospective record review study. BM] Qual Saf. [Internet] 2016 [cited Jan 19, 2018]; 26. Available from: http://dx.doi.org/10.1136/ bmjqs-2015-004828

18. Wilson RM, Michel P, Olsen S, Gibberd RW, Vincent C, El-Assady $R$, et al. Patient safety in developing countries: retrospective estimation of scale and nature of harm to patients in hospital. BM]. [Internet] 2012 [cited Apr 2, 2017]; 344:e832. Available from: https:// doi.org/10.1136/bmj.e832

19. Halfon P, Staines A, Burnand B. Adverse events related to hospital care: a retrospective medical records review in a Swiss hospital. Int J Qual Health Care. [Internet] 2017 [cited Jan 10, 2018]; 29:4. Available from: https://doi.org/10.1093/intqhc/mzx061

20. Kennerly DA, Kudyakov R, Graca B, Saldana M, Compton J, Nicewander D, et al, Characterization of Adverse Events Detected in a Large Health Care Delivery System Using an Enhanced Global Trigger Tool over a Five-Year Interval. Health Serv Res. [Internet] 2014 [cited Jan 19, 2018]; 49:5. Available from: https://doi. org/10.1111/1475-6773.12163

21. Bezerra WR, Bezerra ALQ, Paranaguá TTB, Bernardes MJC, Teixeira CC. Occurrence of incidentes at a surgical center: a documentar stud. Rev Eletron Enferm. [Internet] 2015 [cited Jan 22, 2018]; 17:4. Available from: https://doi.org/10.5216/ree.v17i4.33339

22. Wang $\mathrm{CH}$, Shih $\mathrm{CL}$, Chen WJ, Hung $\mathrm{SH}$, Jhang WJ, Chuang LJ, et al. Epidemiology of medical adverse events: perspectives from a single institute in 
Taiwan. ] Formos Med Assoc. [Internet] 2016 [cited Mar 10, 2018];115(6). Available from: https://doi. org/10.1016/j.jfma.2015.11.004

23. Berríos-Torres SI, Umscheid CA, Bratzler DW, Leas B, Stone EC, Kelz RR, et al. Centers for Disease Control and Prevention Guideline for the Prevention of Surgical Site Infection, 2017. JAMA Surg. [Internet] 2017 [cited Jul 31, 2018];152:8. Available from: https://doi. org/10.1001/jamasurg.2017.0904

24. Roscani ANCP, Ferraz EM, Oliveira Filho AG, Freitas MIP. Validation of surgical checklist to prevent surgical site infection. Acta Paul Enferm. [Internet] 2015 [cited Jan 19, 2018]; 28:6. Available from: http://dx.doi. org/10.1590/1982-0194201500092

25. Fan CJ, PawlikTM, DanielsT, Vernon N, BanksK, Westby $\mathrm{P}$, et al. Association of Safety Culture with Surgical Site Infection Outcomes. ] Am Coll Surg. [Internet] 2016 [cited Jan 19, 2018]; 222:2. Available from: https://doi.org/ 10.1016/j.jamcollsurg.2015.11.008

26. Paranaguá TTB, Bezerra ALQ, Silva AEBC, Azevedo FM Filho. Prevalence of no harm incidents and adverse events in a surgical clinic. Acta Paul Enferm. [Internet] 2013 [cited Jan 22, 2018]; 26:3. Available from: http:// dx.doi.org/10.1590/S0103-21002013000300009

27. Cammarota MC, Ribeiro I Junior, Lima RQ, Almeida CM, Moura LG, Daher LMC, et al. The use of adhesion sutures to minimize the formation of seroma following mastectomy with immediate breast reconstruction. Rev Bras Cir Plást. [Internet] 2016 [cited Jul 31, 2018];31(2). Available from: http://www.dx.doi.org/10.5935/21771235.2016RBCP0026

28. Lopes CMM, Hass VJ, Dantas RAS, Oliveira CG, Galvão $\mathrm{CM}$. Assessment scale of risk for surgical positioning injuries. Rev. Latino-Am. Enfermagem. [Internet] 2016 [cited Feb 22, 2018]; 24: e2704. Available from: http:// dx.doi.org/10.1590/1518-8345.0644.2704

29. Garcia JBS, Bonilla P, Kraychete DC, Flores FC, Valtolina EDP, Guerrero C. Optimizing post-operative pain management in Latin America. Braz J Anesthesiol. [Internet] 2017 [cited Jun 02, 2018]; 67:4. Available from: https://doi.org/10.1016/j.bjane.2016.04.003
Recebido: 10.12.2018

Aceito: 22.03.2019
Autor correspondente:

Josemar Batista

E-mail: josemar.batista@hotmail.com

(D) https://orcid.org/0000-0001-9838-1232
Copyright $\odot 2019$ Revista Latino-Americana de Enfermagem Este é um artigo de acesso aberto distribuído sob os termos da Licença Creative Commons CC BY.

Esta licença permite que outros distribuam, remixem, adaptem e criem a partir do seu trabalho, mesmo para fins comerciais, desde que Ihe atribuam o devido crédito pela criação original. É a licença mais flexível de todas as licenças disponíveis. É recomendada para maximizar a disseminação e uso dos materiais licenciados. 